\title{
Statistical modelling of gluten production by varying mixing time, salt and water levels during dough mixing.
}

\begin{abstract}
Gluten is widely discussed in dough and breadmaking subjects but the focus has not been on the production of gluten itself for usage and application in the food industry. This paper presents some modelling studies of gluten production using response surface methodology (RSM) by varying three main factors that contribute towards gluten formation during dough mixing, which are the mixing time, salt and water levels. The gluten produced was measured for its quantity in terms of wet and dry gluten contents and for quality, in terms of volume expansion of fried gluten and extensibility of gluten. Two wheat flour types, the strong and weak were used as comparison. The experiment was designed following a standard RSM design, known as the central composite design. The results of analysis of variance indicated that the proposed mathematical model obtained can adequately describe the production of gluten within the studied factor limits. The coefficient of determination, R2 of all the responses were higher than 0.80 . Results show that salt gave the most significant effect $(0.0001<\mathrm{P}<0.02)$, followed by water level $(0.0001<\mathrm{P}<0.67)$ and the least significant factor was mixing time $(0.0001<\mathrm{P}<0.95)$.
\end{abstract}

Keyword: Central composite design; Extensibility; Gluten; Response surface methodology; Volume expansion. 